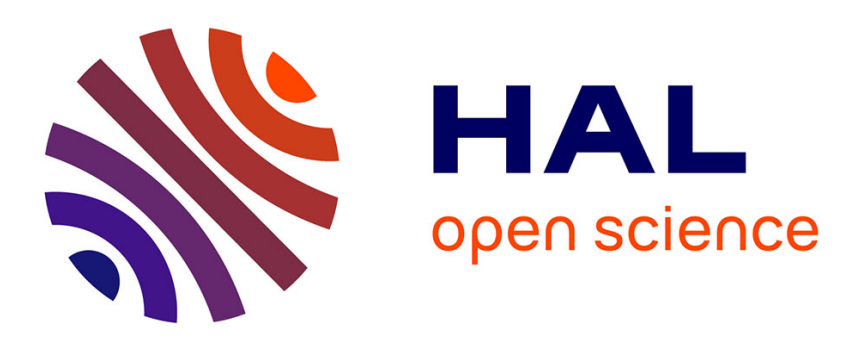

\title{
La régulation éthique de la recherche aux États-Unis : histoire, état des lieux et enjeux
}

François Bonnet, Bénédicte Robert

\section{To cite this version:}

François Bonnet, Bénédicte Robert. La régulation éthique de la recherche aux États-Unis: histoire, état des lieux et enjeux. Genèses. Sciences sociales et histoire, 2009, 2 (75), pp.87-108. 10.3917/gen.075.0087 . halshs-00879312

\section{HAL Id: halshs-00879312 \\ https://shs.hal.science/halshs-00879312}

Submitted on 2 Nov 2013

HAL is a multi-disciplinary open access archive for the deposit and dissemination of scientific research documents, whether they are published or not. The documents may come from teaching and research institutions in France or abroad, or from public or private research centers.
L'archive ouverte pluridisciplinaire HAL, est destinée au dépôt et à la diffusion de documents scientifiques de niveau recherche, publiés ou non, émanant des établissements d'enseignement et de recherche français ou étrangers, des laboratoires publics ou privés.

\section{(ㅇ)(1) $\$$}

Distributed under a Creative Commons Attribution - NonCommercial - NoDerivatives| 4.0 


\title{
La régulation éthique de la recherche aux États-Unis : histoire, état des lieux et enjeux
}

\author{
François Bonnet* et Bénédicte Robert**
}

\begin{abstract}
Résumé. Aux Etats-Unis, toute recherche portant sur des sujets humains est soumise à des comités d'éthique qui peuvent bloquer le financement des projets si ceux-ci contreviennent aux principes éthiques. Les autorités américaines ont été amenées à penser la nécessité d'une régulation éthique à la suite de scandales dans la recherche médicale. Les principes de cette régulation ont été posés en 1979 dans le rapport Belmont, dont les recommandations s'appliquent à toutes les recherches comprenant des sujets humains - y compris par les sciences sociales. La régulation éthique est mise en cuvre par des comités d'éthique décentralisés au niveau de l'université, les Institutional Review Boards. Aucune recherche ne peut être conduite sans leur approbation préalable. Si les chercheurs de sciences sociales s'accordent sur la nécessité de principes éthiques dans la recherche, la critique à l'égard de ces comités s'est durcie ces dernières années. L'histoire de la régulation éthique aux Etats-Unis soulève un certain nombre d'enjeux qui ne sont pas spécifiques au contexte américain.
\end{abstract}

François Bonnet est docteur en sociologie (cotutelle Sciences Po / Université de Milan) et travaille sur les questions urbaines et pénales. Sa thèse a obtenu le prix Gabriel Tarde de l'Association Française de Criminologie. Il est membre du CURP à Columbia University, chercheur postdoctorant à l'OFCE et enseigne à Sciences Po. Il a notamment publié «Un crime sans déviance : le vol en interne comme activité routinière », Revue française de sociologie, vol. $49, \mathrm{n}^{\circ} 2,2008$ et «La distance sociale dans le travail de terrain: compétence stratégique et compétence culturelle dans l'interaction d'enquête », Genèses, n73, 2008.

Agrégée d'anglais et docteure en science politique (Sciences Po, 2007), Bénédicte Robert s'intéresse aux politiques d'éducation dans une perspective comparée. Elle est l'auteur d'une thèse sur l'évolution des politiques de compensation en France et aux Etats-Unis, où elle a conduit trois enquêtes de terrain (Providence, Chicago, Boston), dont l'une en tant que boursière Fulbright. Elle est chargée de mission auprès du recteur de l'académie de Créteil sur les politiques socio-éducatives et chercheure associée à l'OSC.

\author{
* OFCE, Sciences Po et CURP, Columbia \\ University \\ Contact : \\ 52 rue Jeanne d'Arc \\ 75013 Paris \\ francois.bonnet@sciences-po.org
}

\author{
** OSC, Sciences Po \\ Contact : \\ 147 av. Daumesnil \\ 75012 Paris \\ benedicte.robert@sciences- \\ po.org
}




\title{
La régulation éthique de la recherche aux États-Unis : histoire, état des lieux et enjeux
}

\author{
François Bonnet et Bénédicte Robert
}

Comment limiter le risque que le chercheur fasse du mal à ses enquêtés ? Le récent article de Florence Weber (2008) sur les problèmes liés à la publication de cas ethnographiques et la publication du projet de la charte déontologique de l'Association française de sociologie (AFS) ${ }^{\mathrm{i}}$ en mars 2009 montrent l'actualité de la question. En France, pendant longtemps, « l'éthique était censée être incarnée par l'anthropologue ou le sociologue, dont l'intégrité morale et la rigueur scientifique étaient des garanties suffisantes du respect de l'éthique » (Fassin 2006 : 523). La régulation éthique de la recherche qualitative, entendue comme contrôle extérieur de la bienséance d'une recherche donnée, y est minimale voire inexistante. L'idée d'une régulation éthique commune à l'ensemble de la recherche française a été adoptée puis écartée. Seuls les chercheurs français évoluant à l'étranger, et donc soumis aux contraintes locales, sont confrontés à la régulation éthique. Suite à un avis du Conseil national consultatif d'éthique (1993), la loi Huriet de 1988 a été étendue en 1994 aux recherches sur le comportement humain ${ }^{\mathrm{ii}}$. Avant que l'AFS ne publie sa charte - qui n'a pas (encore) de valeur contraignante - la Société française de psychologie était la seule association professionnelle de sciences sociales à avoir adopté, en 2003, un «code de conduite des chercheurs dans les sciences du comportement ». En revanche, aux États-Unis, au Royaume-Uni, au Canada, au Danemark, en Afrique du Sud, les chercheurs ne sont pas entièrement libres de nuire à leurs sujets : ils doivent expliciter et formaliser leur respect de principes éthiques.

Dans les universités américaines, toute recherche qui comprend l'étude de «sujets humains » doit passer devant un comité d'éthique afin d'être autorisée. Si un projet de recherche est rejeté au motif qu'il présente des risques pour les enquêtés, la recherche ne peut pas être financée et le chercheur n'est pas censé pouvoir la publier. Cette disposition est valable pour les sciences médicales comme pour les sciences sociales. Les projets de recherche sont donc soumis au contrôle de comités d'éthique locaux appelés «Institutional Review Boards » (IRB) ${ }^{\text {iii }}$. Cette disposition affecte en premier lieu la recherche qualitative, car le codage systématique des données dans les recherches quantitatives garantit leur confidentialité et minimise les risques pour les sujets aux yeux des IRB ${ }^{\text {iv }}$. Ceux-ci font l'objet d'une controverse qui s'amplifie dans la sociologie américaine. Ils sont accusés de limiter la liberté d'expression et d'être une nouvelle forme de censure au nom de l'éthique.

Ce travail fait le point sur la régulation éthique aux États-Unis. Les autorités américaines ont été amenées à penser la nécessité d'une régulation éthique à la suite de scandales principalement dans la recherche médicale. L'élaboration de la régulation fait suite au rapport Belmont, dont les recommandations s'appliquent pour toutes les recherches impliquant des sujets humains - y compris les sciences sociales. La régulation éthique est mise en œuvre par des comités d'éthique décentralisés au niveau de l'Université, les IRB, qui jugent de la bienséance éthique de projets de recherche (et non de résultats). L'émergence de la régulation éthique pose des problèmes spécifiques pour les sciences sociales.

\section{Les scandales à l'origine de la régulation éthique}

Les succès de la médecine scientifique expérimentale à la fin du XIX ${ }^{\mathrm{e}}$ siècle avaient favorisé dans le monde occidental un contexte d'optimisme scientiste et de foi en la médecine. En 1945, la découverte des expériences médicales réalisées par les médecins nazis sur les prisonniers des camps de concentration bouleverse cet optimisme. En 1966, un professeur d'anesthésiologie de Harvard, Henry K. Beecher, dénonce les pratiques de vingt-deux recherches publiées dans des grandes revues scientifiques (Beecher 1966). La prise en compte des questions éthiques dans la recherche scientifique commence avec la médecine puis s'étend aux sciences sociales. 
En 1932, le Service de santé publique des États-Unis ${ }^{\mathrm{v}}$ lance une recherche ambitieuse sur la syphilis dans le Sud, à Macon County en Alabama. La syphilis est une maladie sexuellement transmissible à l'origine de paralysies, de démence et de crises cardiaques. Dans l'Alabama rural de la Grande Dépression, la plupart des Noirs sont des métayers pauvres et analphabètes, qui ne connaissent de la syphilis que sa désignation vernaculaire, bad blood, le mauvais sang. Au début des années 1930, les usages médicaux de la pénicilline n'ont pas été découverts; les traitements disponibles pour la syphilis sont coûteux, douloureux et aléatoires. Plus de six cents métayers noirs sont réunis par les médecins du Tuskegee Institute pour étudier le développement et la progression de la syphilis. Environ deux cents Noirs qui ne sont pas atteints de syphilis servent de groupe témoin; les quatre cents autres, malades, sont l'objet véritable de l'étude : puisque les traitements disponibles sont si mauvais, quels sont les effets de la syphilis chez des malades non traités ? La durée initialement prévue de l'étude est de six à douze mois ${ }^{\mathrm{vi}}$. En fait, elle va se poursuivre pendant quarante ans, jusqu'au début des années 1970.

Le premier élément problématique de l'étude de Tuskegee est que les Noirs pauvres syphilitiques n'ont jamais été informés qu'ils étaient atteints de cette maladie. Ils ont toujours cru qu'ils avaient le bad blood; les «traitements» qu'ils recevaient étaient des placebos. Non seulement les sujets n'étaient pas traités, mais ils étaient tellement ignorants du protocole de recherche qu'ils croyaient bénéficier d'un traitement de faveur, puisqu'ils recevaient des repas chauds et des «examens médicaux » gratuits, ainsi qu'une concession gratuite pour leur enterrement (en échange du droit de pratiquer une autopsie sur les cadavres). Le deuxième élément problématique est qu'en 1942 des chercheurs d'Oxford découvrent les usages thérapeutiques de la pénicilline, laquelle est immédiatement utilisée, notamment pour les blessés de la Seconde Guerre mondiale. En 1947, la pénicilline devient le traitement standard pour la syphilis. Mais les médecins de Tuskegee n'informent pas leurs patients de cette découverte et conservent le même protocole de recherche jusqu'à la révélation du scandale dans la presse en 1972.

En somme, les médecins (blancs) de Tuskegee voulaient savoir ce qu'il advenait des malades (noirs) de la syphilis quand celle-ci n'était pas traitée, compte tenu de l'absence de traitements satisfaisants ; ils ont trompé leurs patients sur les finalités des examens, qui impliquaient notamment une ponction lombaire extrêmement douloureuse, ne les ont même pas informés de leur maladie réelle ainsi que des conditions de sa transmission, puis ont délibérément tenu ces patients à l'écart du traitement par antibiotiques quand celui-ci est devenu disponible. Pendant vingt-cinq ans, ils ont laissé leurs patients mourir d'une maladie dont ils connaissaient le remède. Sur les quatre cents participants, vingt-huit décèdent directement de la syphilis, cent de complications liées à la maladie, quarante épouses ont été infectées, et dix-neuf enfants sont nés avec la syphilis congénitale. Le directeur du Service de santé publique à Tuskegee entre 1943 et 1948 déclare en 1976 : « le statut de ces hommes ne requérait pas un débat éthique. C'étaient des sujets, pas des patients; du matériel clinique, pas des malades »(Jones $1981: 179)$.

Dans la culture américaine, Tuskegee est devenu synonyme de «science raciste et meurtrière ». Le scandale est d'autant plus vif qu'il existe une longue histoire de l'utilisation des esclaves noirs pour des expériences médicales aux XVIII ${ }^{\mathrm{e}}$ et XIX ${ }^{\mathrm{e}}$ siècles (Savitt 1982). Il semble que les choses sont en fait plus complexes. L'anthropologue Richard Shweder (2004) - dont l'agenda déclaré est de combattre la régulation des sciences sociales par les IRB - a réexaminé l'étude de Tuskegee et apporte un regard plus nuancé. D'une part, il n'apparaît pas absurde de cacher le véritable statut de la maladie (syphilis au lieu de bad blood) aux sujets et à leurs proches, dans la mesure où les mœurs très conservatrices du Sud rural auraient stigmatisé les participants. D'autre part, la syphilis est une maladie dont beaucoup de malades ne souffrent que de façon latente, sans développer de symptômes. Les cas mortels surviennent généralement dans les mois qui suivent l'infection. Ainsi, à la découverte de la pénicilline dans les années 1940, les sujets de Tuskegee qui n'étaient pas morts avant n'avaient qu'une probabilité très faible d'être affectés par les symptômes de la syphilis et le traitement à la pénicilline ne s'imposait pas de façon urgente. L'étude de Tuskegee est peut-être moins abominable qu'elle ne le semble au premier abord; selon Shweder, le contexte de lutte contre le racisme et d'affirmation des droits des Noirs des années 1970 a empêché tout examen serein des 
faits - d'ailleurs, le procès n'a pas eu lieu, l'État fédéral étant parvenu à un accord financier avec les plaignants.

Le fond de l'étude de Tuskegee était de mieux connaître l'évolution d'une maladie en l'absence de traitement. Les médecins de l'hôpital public de Staten Island à New York se sont posé la même question par rapport à l'hépatite. Entre 1963 et 1966, ils ont délibérément infecté un groupe d'enfants handicapés mentaux de l'école publique de Willowbrook ${ }^{\text {vii }}$. Le système public de santé n'offre alors que peu de solutions pour les enfants handicapés mentaux. Les parents se voient offrir l'opportunité de soins gratuits pour leurs enfants en échange de leur accord pour la participation à des expériences - dont la nature exacte reste secrète, et pour cause. Quand le scandale est révélé, les médecins se défendent en prétextant que les conditions sanitaires rendent les infections quasiment inéluctables et qu'il est donc préférable de prendre les devants ${ }^{\text {viii }}$. Comme dans le cas de Tuskegee, les médecins ont tiré parti de la faiblesse, de l'ignorance, de la pauvreté et de l'incapacité à se défendre de leurs sujets (Rothman 1982 ; Rothman et Rothman 1984).

Il existe de nombreux autres exemples d'expériences médicales controversées qui ont eu un impact sur la création des IRB. Entre 1944 et 1974, de nombreuses études ont été réalisées sur les conséquences de l'exposition aux radiations. Des soldats ont marché dans le désert peu après l'explosion d'une bombe atomique, soixante-sept prisonniers des pénitenciers d'État de l'Oregon et de Washington se sont vus irradier leurs testicules et cinquante-quatre enfants handicapés mentaux ont été nourris avec des céréales radioactives. Ces différentes affaires se sont déroulées dans le contexte du mouvement pour les droits civiques des années 1960-1970. Les atteintes de la recherche médicale contre les minorités ont donc été particulièrement scandaleuses. Mais les questionnements sur l'éthique de la recherche ne se sont pas limités à la médecine; les sciences sociales ont été concernées, en raison de pratiques elles aussi douteuses.

\section{Les scandales dans les sciences sociales : Milgram, Zimbardo et Humphreys}

Dans les sciences sociales, plusieurs recherches ont montré des limites éthiques. La plus connue est celle de Stanley Milgram à Yale à partir de 1961. À la suite du procès d'Adolf Eichmann à Jérusalem, Milgram (1994) voulait mesurer l'obéissance à l'autorité. Le protocole de recherche est bien connu : un acteur joue le rôle du faux cobaye qui doit répondre à des questions; le vrai cobaye, qui ne sait rien du protocole et croit participer à une expérience sur la mémorisation, est chargé d'administrer des décharges électriques à chaque fois que le faux cobaye se trompe, tout cela sous l'œil de Milgram impassible. Les décharges électriques sont fausses, et l'acteur feint la douleur, mais le (vrai) cobaye ne le sait pas, et croit qu'il administre de vraies décharges à un vrai cobaye. Chaque erreur implique d'augmenter l'intensité des décharges électriques, jusqu'au maximum de 450 volts (intensité pour laquelle il est écrit sur le panneau de contrôle du vrai cobaye "potentiellement mortel »). Malgré les hurlements de douleur de l'acteur, entre 61 et $66 \%$ des sujets délivrent de leur plein gré la décharge électrique maximale. Aucun des $35 \%$ restant n'essaie de mettre fin à l'expérience ${ }^{\text {ix }}$. Durant les expériences, les sujets de Milgram ont montré de hauts niveaux de stress : rires nerveux inextinguibles, pleurs, sudations, etc. Harvard a refusé de titulariser Milgram en raison des problèmes éthiques de sa recherche.

Dix ans après le début des expériences de Milgram, Philip Zimbardo, le psychologue de Stanford, a voulu analyser les relations entre gardiens et détenus dans les prisons (Haney, Banks et Zimbardo 1973 ; Zimbardo 2007). Il sélectionne par petites annonces vingt et un participants blancs et middle class pour leur stabilité mentale et leur bonne santé. Les participants sont aléatoirement divisés en prisonniers et gardiens. Zimbardo joue le rôle du directeur de la prison, qui est recréée dans les soussols de Stanford. Les gardiens ont pour instruction de maintenir l'ordre sans recourir à la violence physique. L'expérience est censée durer quatorze jours. Dès le deuxième jour, une émeute survient, réprimée à l'aide d'extincteurs. Très rapidement, le groupe des gardiens se partage en trois catégories : les indifférents, les compatissants et les sadiques. Les gardiens sadiques imposent des punitions arbitraires, empêchent les prisonniers d'aller aux toilettes, les forcent à laver les cellules à main nue, à faire des pompes, à chanter, à rester nu un sac sur la tête, etc. Le quatrième jour, Zimbardo envisage de transférer les détenus dans une vraie prison désaffectée et rapporte qu'il est lui-même furieux que la police lui oppose un refus. Le sixième jour, après que près de cinquante 
personnes extérieures ont pris connaissance des conditions de l'expérience sans protester, une étudiante en thèse s'indigne et Zimbardo arrête l'expérience - au grand dam du tiers de gardes sadiques, qui insistent pour continuer à humilier les prisonniers, lesquels sont passés rapidement de la révolte à l'apathie. Les prisonniers et certains gardes sont traumatisés.

Les expériences de psychologie sociale sont présentées de façon routinière aux étudiants comme des grands succès des sciences sociales; c'est moins le cas du travail de Laud Humphreys sur les rencontres homosexuelles dans les toilettes publiques. Humphreys (2007) était en 1961 un doctorant qui voulait rendre compte de l'écart entre normes affichées et comportement effectif. Sa thèse se fonde sur l'observation in situ des relations homosexuelles dans les toilettes publiques pour hommes. Humphreys gagne la confiance de certains participants, devient watchqueen (guetteur), un rôle important puisque la police procède régulièrement à des arrestations. Il décrit avec minutie les signes et les étapes par lesquels les usagers des toilettes montrent leur envie et leur disponibilité. Lui-même homosexuel marié (et prêtre épiscopalien), Humphreys veut en savoir plus sur le mode de vie des hommes qui ont recours aux relations impersonnelles dans les toilettes publiques. Il relève les plaques d'immatriculation des participants et les prend en filature pour savoir où ils habitent. Il espionne ses sujets et va les interroger en se faisant passer pour un employé du Service de santé publique pour recueillir des informations personnelles. Il établit entre autres que $54 \%$ de ses sujets sont mariés. Humphreys ne recevra jamais son doctorat. La presse fustige une recherche attentatoire au respect de la vie privée; l'université ne veut pas cautionner les manquements éthiques, qui provoquent un tort important à la réputation des sciences sociales.

Ces scandales ont inspiré une première réflexion sur les questions éthiques dans les sciences sociales (Kelman 1968; Beauchamp 1982; Stark 2007) et ont abouti en 1971 à l'adoption de codes de déontologie par l'American Sociological Association et l'American Anthropological Association.

\section{L'institutionnalisation de la régulation éthique (1966-1991) : la common rule}

À partir des années 1960, le gouvernement fédéral commence à s'intéresser aux questions éthiques, en particulier dans les recherches qu'il finance. Les travaux menés par le Service de santé publique et le Congrès américain donnent naissance à la common rule, qui constitue aujourd'hui le cadre éthique législatif et réglementaire de la recherche aux États-Unis. La common rule concerne toutes les recherches sur les sujets humains (médecine et sciences sociales) financées par un contrat de recherche avec l'administration fédérale.

\section{Le rapport Belmont : les principes de la régulation éthique}

En 1966, dans un contexte d'augmentation continue du financement fédéral de la recherche, le Service de santé publique constate les insuffisances des codes de déontologie adoptés par les diverses organisations professionnelles. Il met en place les premiers comités d'éthique, les review committees. Dès cette époque, les compétences de ces comités d'éthique s'étendent aux sciences sociales et à la psychologie : les National Institutes of Health, qui fournissent $20 \%$ du budget fédéral pour la recherche, financent en effet d'importantes recherches en sciences sociales, notamment par le biais de l'Institut national de santé mentale et de l'Institut national des sciences médicales ${ }^{\mathrm{x}}$. Ce type de financements illustre la proximité entre les sciences sociales et les sciences médicales aux ÉtatsUnis (voir Weber 2008). Les contrats de recherche du gouvernement fédéral (à l'origine de la régulation éthique) ont rapproché les recherches sur les sujets humains. Les comités d'éthique sont rapidement étendus à l'ensemble de la recherche financée par le ministère de la Santé et des Affaires sociales $^{\mathrm{xi}}$ dont dépend le Service de santé publique. Ces comités sont locaux, décentralisés au niveau de l'Université, afin de désengager la responsabilité des principaux financeurs publics, tels les National Institutes of Health (Stark 2007).

Cette organisation décentralisée donne lieu à des écarts d'interprétation incitant le Service de santé publique à préciser sa démarche. En mai 1974, le ministère publie l'arrêté 45CFR46, qui est toujours en vigueur (Department of Health and Human Services 2005). Cet arrêté précise qu'aucune 
recherche ne pourra être financée par le ministère sans l'approbation d'un IRB. L'arrêté s'inspire largement des travaux entrepris au Service de santé publique ; il sert de base à la loi nationale sur la recherche (1974) ${ }^{\mathrm{xii}}$. Cette loi crée aussi la Commission nationale pour la protection des sujets humains impliqués dans la recherche biomédicale et du comportement humain ${ }^{\text {xiii }}$. La commission doit suivre la mise en œuvre des IRB, rendre des avis concernant la recherche sur les populations les plus vulnérables (enfants, prisonniers, femmes enceintes, handicapés) et surtout identifier les principes éthiques communs à l'ensemble de la recherche sur les sujets humains. En 1979, sept ans après le scandale de Tuskegee, la commission publie le «rapport Belmont» (The National Commission for the Protection... 1979) ${ }^{\mathrm{xiv}}$ qui énonce les trois principes éthiques sur lesquels la recherche sur les sujets humains doit se fonder :

- Principe de respect : la recherche porte sur des sujets humains autonomes et, le cas échéant, doit protéger les personnes vulnérables ;

- Principe de bienfaisance : la recherche ne doit pas nuire aux sujets ;

- Principe de justice: la recherche ne doit pas désavantager systématiquement certaines catégories de personnes.

Ces principes sont suivis de recommandations insistant sur l'importance du consentement éclairé (informed consent), de la réflexion sur l'équilibre entre les bénéfices et les risques de la recherche (benefit-risk ratios), ainsi que de l'équité du mode de sélection des sujets de la recherche.

\section{De la contestation initiale à la formation d'un consensus}

Sur la base des recommandations du rapport Belmont, le Service de santé publique publie une proposition de modification de l'arrêté 45CFR46 de 1974 qui instituerait une régulation éthique extrêmement rigoureuse pour toutes les recherches sur les sujets humains. Les chercheurs en sciences sociales s'insurgent contre cette proposition. Ithiel de Sola Pool, politiste au MIT (Massachusetts Institute of Technology), invite les chercheurs qui le souhaitent à le rejoindre dans un "Comité de vigilance sur les pratiques des IRB ${ }^{\mathrm{xv}}$ dont le manifeste explique que le projet de révision envisagé va à l'encontre de la liberté d'expression garantie par le Premier amendement à la Constitution ; le contrôle préalable des IRB est comparable, selon lui, à de la censure (Pool 1979). Il est soutenu par de nombreux chercheurs, dont Gabriel Almond, Nathan Glazer, Charles Lindblom, Laud Humphreys et George Homans (Social Scientists Form Committee to Protest Proposed Regulations 1979).

En réponse, le ministère de la Santé et des Affaires sociales publie en 1981 un arrêté en retrait de la proposition initiale. Cette décision est bien accueillie par les chercheurs de sciences sociales (Citro, Ilgen et Marrett 2003 : 73). En 1991, quinze autres agences et ministères s'associent à ce dispositif en adoptant les règles posées en 1981, désormais connues comme la common rule, commune à l'ensemble des disciplines et des laboratoires bénéficiant de contrats de recherche fédéraux. La révision de 1991 accroît le nombre de contrats de recherche couverts par la réglementation fédérale en matière d'éthique, assurant ainsi la protection d'un plus grand nombre de sujets humains. Les laboratoires et les départements doivent s'engager par écrit à se conformer à cette règle (assurance of compliance), sans quoi ils ne peuvent bénéficier des contrats de recherche fédéraux. Les IRB sont chargés de la mise en œuvre du dispositif. Aucune recherche ne peut être entamée sans leur validation.

À la fin des années 1990, les IRB gèrent avec des moyens relativement constants un nombre de plus en plus important de dossiers, en partie à cause de la forte augmentation des recherches financées par l'industrie pharmaceutique. Face à l'engorgement des IRB, une série de rapports pointent les insuffisances du dispositif (Department of Health and Human Services. Office of Inspector General 1998 ; United States General Accounting Office 1996; United States. Advisory Committee on Human Radiation Experiments 1995). Le rapport de la Commission nationale consultative sur la bioéthique $^{\mathrm{xvi}}$, nommée en 1995 par le président Clinton, confirme les constats établis par les différentes branches de l'administration (National Bioethics Advisory Commission 2001). En réponse, les autorités fédérales intensifient leurs inspections des IRB et vont jusqu'à prononcer la fermeture temporaire des laboratoires. À la suite d'une inspection, le centre médical de l'Université de Duke est fermé pendant cinq jours (Greenberg 1999, 2001 ; Holden 1999) ; en 2001, toutes les 
recherches à Johns Hopkins sont interrompues à la suite de la mort d'une jeune femme (pourtant en bonne santé) au cours d'une étude sur l'asthme. Dans ce contexte d'augmentation de la recherche et de surveillance accrue par le gouvernement fédéral, les IRB font preuve d'un «excès de zèle » (Marshall $2004: 270$ ), notamment à l'égard des recherches en sciences sociales, créant un «climat réglementaire » relativement nouveau (Plattner $2003: 290)$. En conséquence, le débat évolue : il ne porte plus sur les principes de la régulation éthique, mais sur la mise en œuvre.

\section{Le fonctionnement des IRB : la mise en ouvre de la common rule}

La décentralisation de la régulation éthique est la source principale des problèmes de mise en œuvre de la common rule. Les IRB disposent d'une marge d'interprétation considérable (Bell, Whiton et Connelly 1998; Citro, Ilgen et Marrett 2003; De Vries et Forsberg 2002 ; Gray, Cooke et Tannenbaum 1978 ; Hayes, Hayes et Dykstra 1995 ; Sieber et Baluyot 1992), comme en témoignent la variabilité de leurs décisions à propos d'un même protocole ainsi que la durée de la délibération (Goldman et Katz 1982 ; Rogers et al. 1999 ; Silverman, Hull et Sugarman 2001) ${ }^{\text {xvii }}$. L'appréciation du risque encouru par les sujets est ainsi particulièrement variable.

\section{Une définition variable des risques et des protections}

L'arrêté 45CFR46 attribue aux IRB le droit d'«exempter» certaines recherches d'examen, de procéder à un examen «accéléré » (expedited review) du protocole de recherche, ou d'accorder des dérogations (waivers) et définit le «risque minime» comme un niveau de risque «que l'on peut rencontrer dans la vie de tous les jours ». Il existe un consensus interdisciplinaire sur le fait que la plupart des recherches en sciences sociales et du comportement ne posent, selon les termes de l'arrêté, qu'un «risque minime » aux sujets humains de la recherche (Bell, Whiton et Connelly 1998 ; Department of Health and Human Services. Office of Inspector General 1998, 2000 ; Gordon 2003 ; Hayes, Hayes et Dykstra 1995; Marshall 1992a, 1992b, 2004 ; United States General Accounting Office 1996). Le texte de l'arrêté concernant les recherches pouvant être exemptées semble en effet couvrir l'essentiel des méthodes de recherche des sciences sociales :

\footnotetext{
«[Sont passibles d'examen accéléré ou de dérogations] les recherches impliquant l'utilisation de questionnaires, d'entretiens ou d'observation de comportements dans les lieux publics, sauf si : (i) l'information recueillie est enregistrée de telle façon que les sujets humains peuvent être identifiés, directement ou à travers des indices ; et (ii) la divulgation des réponses du sujet humain à l'extérieur du champ de la recherche peut entrainer des risques de type pénal ou civil pour le sujet ou nuire à son statut financier, à son employabilité ou à sa réputation. »
}

Cette définition laisse aux IRB une grande liberté d'interprétation. Pour satisfaire à la première clause, ils peuvent demander à ce que les enregistrements audio des entretiens soient retranscrits et anonymés, puis détruits (Gordon 2003). L'indemnisation des sujets de recherche (une pratique courante aux États-Unis) est parfois compliquée, car certains IRB considèrent que les formulaires d'indemnisation peuvent permettre d'identifier ces sujets. Pour satisfaire à la seconde clause, les chercheurs ont de plus en plus recours à un « certificat de confidentialité » par lequel ils s'engagent à ne pas divulguer d'information portant sur un sujet de la recherche, dans quelque procédure que ce soit (civile, pénale, administrative, législative ou autre) et à quelque niveau que ce soit (fédéral, étatique ou local) (Gordon 2003).

Dans ce débat, l'une des dimensions procédurales qui a le plus cristallisé les discussions est celle du « consentement éclairé » par lequel un individu accepte, en connaissance de cause, de participer à une recherche. Ce consentement peut être écrit ou verbal. Dans les études précédemment citées insistant sur la variabilité des réponses des IRB, les auteurs indiquaient que les ceux-ci étaient plus 
ou moins exigeants en matière de consentement éclairé, certains IRB se contentant d'un consentement verbal. D'autres au contraire exigent, avant d'approuver le protocole de recherche permettant au chercheur de commencer l'enquête de terrain, autant d'exemplaires signés du consentement que de sujets humains impliqués dans la recherche, le texte du consentement ayant été soumis au préalable à l'IRB. Les exigences des IRB peuvent aller jusqu'à demander un calendrier des entretiens et observations :

«Les IRB sont généralement réticents à ne pas demander de consentement éclairé écrit. De plus, les IRB peuvent exiger une formulation peu claire ou peu pertinente pour les sujets potentiels de la recherche. Malgré des recommandations portant sur la compréhension et la lisibilité des formulaires de consentement, certains IRB exigent d'inclure des formulations écrites dans un anglais de niveau lycée ou même licence » (Marshall $2003: 275$ ).

Dans les sciences sociales, la question du consentement éclairé est le principal facteur qui retarde l'approbation du protocole de recherche par l'IRB. En effet, les IRB ont tendance à privilégier le consentement écrit et à ne pas considérer la demande de consentement comme un «acte de communication» (Marshall 2003). Cette démarche est particulièrement problématique dans les recherches sur les comportements illégaux (maltraitance d'enfants, utilisation de drogues, prostitution), sur les enfants, ou sur les personnes illettrées ou avec un faible niveau d'alphabétisation.

\section{Une mise en cuvre des procédures pusillanime}

Dans la mesure où l'essentiel de la recherche en sciences sociales est théoriquement exempté d'examen par un IRB (ou sujet à un examen accéléré), l'augmentation du nombre de protocoles devant être examinés par les IRB au cours les années 1990 aurait pu être une incitation à recourir à l'exemption ou à l'examen accéléré pour les recherches de risque minime. Toutes les études montrent que tel n'a pas été le cas : selon l'étude quantitative la plus récente sur le fonctionnement des IRB (Bell, Whiton et Connelly 1998), la moitié seulement des protocoles pouvant être exemptés l'ont effectivement été.

Bien que les recherches en sciences sociales ne menacent jamais l'intégrité physique des sujets, la plupart des IRB ont décidé d'examiner les projets de recherche avant de prononcer une éventuelle exemption (Katz 2006). En théorie, la composition des IRB doit respecter un principe de diversité et de représentativité $e^{\text {xiii }}$; on observe cependant que les représentants des sciences sociales sont sousreprésentés dans les IRB, ce qui complique la situation (Marshall 2003 ; De Vries et Forsberg $2002)^{\mathrm{xix}}$.

Les IRB ont adopté une démarche excessivement prudente, se traduisant par une extension des recherches placées sous leur surveillance, par crainte de voir le laboratoire ou l'université sanctionnés par les autorités fédérales (Dingwall 2007; Feeley 2007a; Katz 2007). Ils ont par exemple inclus dans leurs missions les recherches financées par le secteur privé, ainsi que les recherches ne faisant pas l'objet d'un contrat de recherche (American Association of University Professors 2001). De manière générale, les IRB ont eu tendance à «gonfler le niveau perçu de risque » (Gordon $2003: 302)$ :

«Au cours de cette étude, les présidents des IRB ont indiqué que pour tout protocole de recherche pouvant être exempté ou examiné rapidement, une proportion importante des IRB - allant de 25 à $77 \%$, en fonction du volume traité par l'IRB et de la catégorie de recherche - choisit comme pratique de référence un examen plus rigoureux que celui préconisé dans l'arrêté » (Bell, Whiton et Connelly 1998 : 77). 
Or, les IRB engorgés ont moins de ressources pour rendre leur décision et sont plus à même de faire des erreurs (Department of Health and Human Services. Office of Inspector General 1998, 2000 ; United States General Accounting Office 1996). En conséquence, la régulation éthique soulève un certain nombre de problématiques.

\section{Les enjeux pour la recherche en sciences sociales}

Trois problématiques spécifiques aux sciences sociales sont particulièrement explorées par les auteurs qui ont travaillé sur la question des IRB : les problèmes liés au rapport au journalisme, à la tromperie (deception) et les problèmes spécifiques liés à l'ethnographie.

\section{Les recherches undercover et la concurrence du journalisme}

Les recherches undercover sont celles où le chercheur dissimule son identité à ses enquêtés et se fait passer pour «un des leurs » afin de produire des données qui n'auraient pas pu exister avec un dispositif d'enquête plus frontal. La sociologue Monica McDermott (2006) s'est par exemple fait embaucher comme caissière dans des supermarchés pour comparer les relations de classe et de race à Atlanta et à Boston; elle a montré comment les Blancs du Sud développent une identité raciale (en opposition aux Noirs) tandis que les Blancs du Nord-Est ont une identité ethnique (Italiens, Irlandais, WASP [White Anglo-Saxon Protestant], etc.). Son travail a été bien reçu; elle a été embauchée par Stanford University. Cependant, ce type d'enquête est menacé par certains IRB, qui considèrent que les enquêtés doivent être informés des buts de l'enquête. Or les sciences sociales ne sont pas le seul discours sur le monde social; elles sont en concurrence avec d'autres types de discours qui ne sont pas soumis aux mêmes contraintes éthiques. L'exemple de la journaliste Barbara Ehrenreich est ainsi abondamment cité par les chercheurs hostiles à la régulation éthique (Feeley 2007a; Dingwall 2007).

Barbara Ehrenreich est une journaliste américaine, docteur en biologie et auteur à succès, qui a quitté sa famille et ses amis entre 1998 et 2000 pour vivre comme une working poor. Elle a travaillé dans des restaurants, comme femme de ménage et à Wal-Mart. En 2001, elle publie Nickel and Dimed (Ehrenreich 2001), le compte rendu de son expérience. Elle revendique une critique « de gauche » de la welfare reform de 1996 de Bill Clinton, qui oblige de nombreux bénéficiaires de l'aide sociale à travailler. Elle veut montrer que ce n'est pas la paresse qui empêche les pauvres de réaliser le rêve américain, et que les salaires trop faibles des emplois non qualifiés, conjugués à des loyers trop chers, ne permettent pas de vivre dignement. Son ouvrage est un best-seller que la plupart des étudiants en sociologie des universités américaines doivent lire dans les premières années de leur cursus. Pourtant, il n'est pas sûr qu'un sociologue puisse à terme mener la même recherche. Ses employeurs ne savent pas qu'ils ont embauché une journaliste undercover et n'ont pas eu à signer de formulaire de consentement éclairé. Kevin Haggerty (2004) souligne l'incongruité de la situation: en se liant les mains, les sociologues laissent le champ libre aux journalistes ${ }^{\mathrm{xx}}$.

\section{Le problème de la tromperie délibérée}

Un autre problème est celui de l'usage de la tromperie délibérée (deception) dans les sciences sociales, et tout particulièrement dans la psychologie sociale (domaine de prédilection de Milgram et Zimbardo). Une étude citée par Haggerty (2004) a montré que presque la moitié des articles scientifiques de psychologie sociale publiés entre 1965 et 1985 était fondée sur la tromperie des sujets $^{\mathrm{xxi}}$. Or l'usage de la tromperie est censé être proscrit par les IRB, puisqu'il viole le principe du consentement éclairé. Cela n'a pas empêché la sociologue Devah Pager de mener sa recherche. Pager a réalisé sa thèse sur les discriminations à l'embauche (2002). Elle montre que les Noirs et les anciens détenus ont une probabilité plus faible d'être recrutés (35\% de taux de rappel pour les Blancs sans casier, $5 \%$ pour les Noirs avec casier). Son protocole empirique consiste à répondre à des petites annonces en envoyant des comédiens noirs et blancs avec des faux curriculum vitæ 
identiques et de mesurer le taux de rappel par les employeurs après entretien (Pager 2003). Un protocole similaire avait été utilisé par l'économiste John Yinger (voir une revue de ces méthodes dans Ross et Yinger 2006). Le travail de Pager a été bien récompensé. Elle a obtenu le prix de la meilleure thèse de l'American Sociological Association en 2003, son article dans l'American Journal of Sociology (2003) a été republié dans huit anthologies et elle a été recrutée à Princeton. Or, selon Katz (2007), une recherche similaire a été bloquée par un IRB dans une université de la côte est, en raison de l'absence de consentement éclairé de la part des employeurs. Les travaux de Pager montrent l'intérêt heuristique que revêt l'usage de la tromperie en sciences sociales.

Les testings représentent l'aboutissement de la logique de tromperie dans les sciences sociales : les enquêtés ne savent même pas qu'ils participent à une recherche et toutes les précautions sont prises pour qu'ils ne se rendent pas compte que la recherche vise à les dénoncer. Les expériences de psychologie sociale où les enquêtés savent qu'ils participent à une recherche mais ne savent pas quels en sont les buts réels peuvent être fascinantes et quand la dissimulation des problématiques de recherche porte sur des échantillons interchangeables, le risque paraît négligeable pour les sujets. Cependant, quand la dissimulation a pour objet des individus qui peuvent se reconnaître dans les travaux publiés, a fortiori quand ces sujets ne sont pas anonymés, le cas est plus problématique. La récente controverse autour de l'article sur l'écrivain Victoria Thérame (Naudier 2006 ; Weber 2008) donne à voir le sentiment de trahison et d'exploitation que peuvent ressentir les enquêtés qui ont eu l'impression que la problématique du travail auquel ils ont coopéré de bonne foi ne leur avait pas été pleinement expliquée. Les chercheurs qui font des entretiens savent qu'il faut parfois déguiser leur problématique, en omettre certains aspects (cruciaux) pour obtenir le consentement et la coopération des enquêtés, y compris quand ces derniers sont anonymés. La frontière entre savoir-faire et manipulation peut être ténue. Recourir à des pratiques déloyales risque d'affecter la réputation de la recherche. Dans cette perspective, l'objet de la régulation éthique est de s'assurer de la pérennité de la recherche - pérennité de sa légitimité et donc de ses financements.

\section{La spécificité de l'ethnographie : comment réguler a priori?}

L'un des aspects les plus controversés de l'évaluation ex-ante par les IRB est son inadéquation avec le travail ethnographique. Les ethnographes ne peuvent sérieusement décrire leur protocole de recherche en faisant une évaluation des risques que celui-ci comporte pour les enquêtés, puisque le travail de terrain implique, dans une large mesure, de ne pas savoir de quoi sera fait le terrain. Les relations personnelles qui se nouent un peu au hasard des rencontres et des affinités se prêtent mal aux formulaires de consentement éclairé. Cependant, quand les recherches portent sur les questions de déviance, les dilemmes éthiques sont plus difficiles à résoudre, comme l'illustre le cas de la recherche de Sudhir Venkatesh.

Entre 1989 et 1996, Sudhir Venkatesh, alors étudiant en thèse à l'Université de Chicago, a mené une ethnographie des Robert Taylor Homes, l'un des plus grands complexes de logements sociaux des États-Unis, peuplés à $96 \%$ par des Noirs vivant sous le seuil de pauvreté. Il a publié deux livres universitaires : American Project et Off the Books (Venkatesh 2002a, 2006), qui ont respectivement reçu un prix de l'American Sociological Association et le C. W. Mills Award. En 2008, Venkatesh publie un livre autobiographique, Gang Leader for a Day, sur son expérience auprès du gang des Robert Taylor Homes, les «Black Kings ». Venkatesh veut rendre compte des relations personnelles qu'il a entretenues avec les différents protagonistes du ghetto, de Ms. Bailey, présidente de l'association des locataires, à J. T., le chef des Black Kings, qui supervise le trafic de crack. Il explique la difficulté de l'ethnographe, qui construit son succès professionnel (Junior Fellow à Harvard, professeur à Columbia) «sur le dos » de ses enquêtés, des Noirs pauvres dont le quartier sera bientôt détruit. Dans un article méthodologique, Venkatesh (2002b) avait dévoilé l'un de ses dilemmes éthiques: alors qu'il avait accepté de trouver parmi les étudiants de l'Université de Chicago des clients pour le garagiste clandestin des Robert Taylor Homes, il avait refusé d'en ramener pour les prostituées locales. Ses interlocuteurs n'avaient ni compris ni apprécié cette réticence, ne voyant pas ce qu'il y avait de moins noble dans la prostitution, et lui avait fait vertement savoir, révélant ainsi le différentiel d'appréciation morale de la prostitution entre le mainstream et le ghetto. 
Dans Gang Leader for a Day, Venkatesh montre que les questions éthiques se sont posées de manière plus pressante. Il explique qu'il a pris part au tabassage collectif d'un conjoint violent et qu'il a assisté aux préparatifs d'un drive-by shootingxii , ce qui l'a obligé à consulter un avocat pour se prémunir contre des actions en justice. L'ethnographie recèle une part d'imprévisible qui pose un problème pour la régulation éthique a priori. Parce que la régulation a priori leur semble insatisfaisante, Mitchell Duneier (1999) et Nicole Marwell (2007) recommandent aux ethnographes de faire valider par les enquêtés les citations qui leur sont attribuées et de ne pas anonymer les interviewés ${ }^{x x i i i}$. L'idée est double : il s'agit d'une part de responsabiliser les chercheurs, qui ne pourront pas se réfugier derrière l'anonymat de leurs enquêtés pour évacuer certaines questions éthiques et, d'autre part, de permettre la réplication des enquêtes afin de réduire le déficit de légitimité épistémologique du travail de terrain. Ces positions ne font pas l'unanimité, comme le montre la typologie de la perception des IRB dans les sciences sociales américaines.

\section{Une typologie de la perception des IRB dans les sciences sociales américaines}

On peut distinguer trois positions dans la communauté des sciences sociales américaines sur la question des IRB.

- La position «critique » dénonce l'émergence d'une censure au nom de l'éthique, son caractère arbitraire, l'aseptisation des sciences sociales et la menace qui pèse sur les méthodes qualitatives en général et l'ethnographie en particulier. Parmi ses auteurs, le criminologue canadien Kevin Haggerty (2004), le sociologue de UCLA (University of California, Los Angeles) et auteur de Seductions of Crime Jack Katz (1988, 2006, 2007), l'anthropologue de l'Université de Chicago Richard Shweder (2006), le juriste Malcolm Feeley (2007a, 2007b), l'anthropologue de Princeton Rena Lederman (2006) et l'auteur de Outsiders Howard Becker (1963, 2004). Haggerty, Lederman et Feeley notamment défendent la thèse du mission creep, selon laquelle les IRB suivent la voie de toute bureaucratie de réglementation, c'est-à-dire de s'étendre bien au-delà de son mandat initial. Selon Katz (2006), le principal objectif des administrations des centres de recherche est de désengager leur responsabilité plutôt que de garantir l'éthique de la recherche. La position critique est la plus couramment défendue dans la littérature sur les IRB.

- La position « réaliste » est principalement défendue par la sociologue Laura Stark $(2006,2007)$. Stark a récemment soutenu une thèse à Princeton sur les IRB. Les réalistes considèrent que la régulation éthique est nécessaire, inévitable et finalement peu contraignante et que la position « critique » est déplacée et contre-productive. Stark estime que les chercheurs en sciences sociales ne sont pas des victimes, qu'ils ne gagnent rien à jouer à se faire peur et qu'ils devraient plutôt investir les IRB de façon à sécuriser leurs intérêts. Par contre, Stark (2007) se montre «peu enthousiaste » devant la proposition des maximalistes de pratiquer une régulation éthique ex-post.

- La position «maximaliste» est soutenue par le sociologue Charles Bosk et le chercheur en bioéthique Raymond De Vries. Pour les maximalistes, la régulation ne doit pas se faire a priori sur des projets de recherche, mais a posteriori sur les résultats de ces recherches, c'est-à-dire sur les publications. Pour Bosk et De Vries (2004), le débat sur l'encadrement éthique de la recherche ne peut progresser tant que l'activité des IRB ne sera que prospective, sans suivi (monitoring) de la recherche une fois le protocole approuvéxiv ${ }^{\text {xxiv }}$ Cette question n'est pas nouvelle (Christakis 1988); elle a fait l'objet d'une recommandation dans le rapport Bell. La position maximaliste part du constat que les problèmes éthiques graves proviennent le plus souvent du non respect par les équipes de recherche du protocole approuvé par l'IRB. Les maximalistes ne sont cependant pas des optimistes béats : pour Bosk (2004) et De Vries (2004), l'une des conséquences de la bureaucratisation de la régulation éthique est que la plupart des chercheurs de sciences sociales consacrent plus de temps à régler des problèmes de procédure, plutôt qu'à réfléchir ensemble aux problèmes éthiques fondamentaux qui se posent au cours de la recherche. 
Il convient de préciser qu'il est difficile de trouver des régularités évidentes dans l'adhésion aux positions critique, réaliste ou maximaliste. De même, il faudrait réaliser un sondage pour connaître l'influence relative des différentes positions.

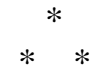

Pour conclure, on peut esquisser deux débats à partir des problématiques de la régulation éthique. Le premier débat porte sur la dépendance relativement nouvelle des chercheurs français à l'égard de certains modes de financement. Aujourd'hui, les contrats de recherche européens, qui représentent une part croissante du budget de la recherche, comportent des exigences accrues en matière éthique, lesquelles sont inexistantes dans les financements nationaux non fléchés. La conjugaison des financements européens avec l'internationalisation des équipes de recherche peut laisser penser que de plus en plus de chercheurs français vont être amenés à expliciter leur positionnement éthique. En surplomb de cette question se pose le débat de l'autonomie scientifique de la recherche. D'un côté, de nombreux chercheurs estiment que l'autonomie absolue de la définition de l'agenda scientifique est une condition sine qua non de la science. En d'autres termes, la question de la liberté du chercheur est de nature épistémologique : on ne peut pas faire de la science quand on est sous contrôle. D'un autre côté, la science coûte cher, a besoin de financements, et les financeurs estiment que leur contribution leur donne un droit de regard minimal, ne serait-ce que pour se protéger d'un scandale potentiel. Dans un contexte de remise en cause des financements publics automatiques et de compétition croissante sur les appels d'offre, l'opposition à la régulation éthique au nom de la liberté scientifique pourrait se traduire par une marginalisation professionnelle - l'éthique de la recherche n'étant qu'un des critères normalisés de scientificité parmi d'autres dans les contrats de recherche.

Le second débat porte sur le rapport des chercheurs à leur travail de terrain. Tous les sociologues qui écrivent contre les IRB affirment en préambule être extrêmement préoccupés par les questions éthiques et par la protection des enquêtés. Il ne faut cependant pas esquiver la question que pose Stark: « les régulations sur les sujets humains ne nous énervent-elles pas pour des raisons moins nobles que la défense de la liberté de la recherche ?»(2007: 785). Peu de chercheurs sont enthousiastes à l'idée de devoir passer par une étape bureaucratique supplémentaire pour pouvoir mener leur enquête de terrain. La plupart des sociologues sont probablement réticents à ce que des pairs ou des évaluateurs extérieurs se penchent sur « la cuisine » du terrain : les enregistrements, les notes brutes, tout ce qui n'a pas été poli et embelli pour publication. Dans cette optique, et c'est le sens de la remarque de Stark, l'hostilité à la régulation éthique est forcément suspecte : elle indique que les chercheurs en sciences sociales ont quelque chose à cacher.

Enfin, au-delà des principes normatifs ou des questions de bonne volonté, la communauté scientifique pourrait appréhender la question selon une approche stratégique. La régulation éthique n'a pas pour unique fin de soulager la conscience des sociologues; c'est aussi un moyen de renforcer la légitimité de la profession. D'un côté, les sciences sociales se doivent d'être performantes en termes de résultats pour justifier de leur utilité et donc de leurs financements. De l'autre, les sciences sociales ont nécessité d'avoir des méthodes que la population juge acceptables : les sociologues travaillent sur des êtres humains et ont besoin de leur coopération. Si les sociologues développent la réputation d'utiliser des méthodes qui nuisent aux sujets, ces derniers n'ont plus aucune raison de coopérer. La légitimité des fins, qui se mesure en termes de pertinence de la connaissance produite, peut s'opposer à la légitimité des moyens, qui renvoie aux méthodes utilisées par les sociologues et se mesure en termes de crédibilité et de réputation. La régulation éthique sert à protéger les sociologues d'eux-mêmes et à crédibiliser leur profession, en s'assurant du concours et de la coopération des sujets; mais une régulation trop sévère risque d'empêcher les sociologues de développer des méthodes innovantes et susceptibles de produire des résultats pertinents. En somme, le débat sur la régulation éthique n'est pas de savoir si celle-ci est opportune ou non, mais de réaliser un double arbitrage : (1) un arbitrage sur l'ampleur de la régulation, entre pertinence (la légitimité des fins) et crédibilité (la légitimité des moyens); et (2) un arbitrage sur la mise en œuvre de cette régulation, ex-ante ou ex-post. 


\section{OUVRAGES CITÉS}

AMERICAN ASSOCIATION OF UNIVERSITY PROFESSORS. 2001. Protecting human beings: Institutional review boards and social science research. Academe, vol. 87, $\mathrm{n}^{\circ} 3$ : 55-67.

BEAUCHAMP, Tom L. (éd.). 1982. Ethical Issues in Social Science Research. Baltimore, Johns Hopkins University Press.

BECKER, Howard S. 1963. Outsiders. Studies in the Sociology of Deviance. New York, Free Press.

— 2004. «Comment on Kevin D. Haggerty, "Ethics Creep : Governing Social Science in the Name of Ethics" », Qualitative Sociology, vol. 27, n $4: 415-416$.

BEECHER, Henry K. 1966. «Ethics and Clinical Research ». New England Journal of Medicine, $\mathrm{n}^{\circ} 274$ : 1354-1360.

Bell, James, John Whiton et Sharon ConNElly. 1998. Evaluation of NIH Implementation of Section 491 of the Public Health Service Act, Mandating a Program of Protection for Research Subjects. Bethesda, MD, National Institutes of Health.

BERNARD, Claude. 1865. Introduction à l'étude de la médecine expérimentale. Paris, J. B. Baillière et fils. BosK, Charles L. 2004. "The Ethnographer and the IRB. Comment on Kevin D. Haggerty, "Ethics Creep : Governing Social Science in the Name of Ethics” », Qualitative Sociology, vol. 27, $n^{\circ} 4$ ) : 417420.

— et Raymond G. DE VRIES. 2004. «Bureaucracies of Mass Deception : Institutional Review Boards and the Ethics of Ethnographic Research », Annals of the American Academy of Political and Social Science, vol. 595, $\mathrm{n}^{\circ} 1: 249-263$.

BOURMEAU, Sylvain. 1988. «Journaliste et sociologue. Robert Ezra Park et la presse », Politix, $\mathrm{n}^{\circ}$ 3-4 : 50-61.

CEFAÏ, Daniel. 2009. «Codifier l'engagement ethnographique ? Remarques sur le consentement éclairé, les codes d'éthique et les comités d'éthique », http://www.laviedesidees.fr/Codifier-l-engagement.html ChrISTAKIS, Nicholas A. 1988. «Should IRBs Monitor Research More Strictly?», IRB: Ethics and Human Research, vol. 10, $\mathrm{n}^{\circ} 2: 8-10$.

Citro, Constance F., Daniel R. Ilgen et Cora B. MARrett (éd.). 2003. Protecting Participants and facilitating social and behavioral sciences research. Washington, DC, National Academies Press.

COMITE CONSUltatif NATIONAL D'ETHIQUE (CCNE). 1993. Avis sur l'éthique de la recherche dans les sciences du comportement humain. (Avis $\left.\mathrm{n}^{\circ} 38\right)$.

COMITE D'ETHIQUE (COMETS). 2007. Réflexions sur éthique et sciences du comportement humain. Paris, CNRS.

DE VRIES, Raymond G. et Carl P. Forsberg. 2002. «What Do IRBs Look Like? What Kind of Support Do They Receive ? », Accountability in Research : Policies et Quality Assurance, vol. 9, n³-4 : 199-216.

Department of Health AND Human Services. 2005. «Protection of Human Subjects », Code of Federal Regulations, vol. 45, partie 46.

DePartment of Health AND Human SERVICES. OfFICE OF InSPECTOR GENERAL. 1998. Institutional Review Boards : A Time for Reform (OEI-01-97-00193). Department of Health and Human Services, Office of Inspector General.

- 2000. Protecting Human Research Subjects: Status of Recommendations (OEI-01-97-00197). Department of Health and Human Services, Office of Inspector General.

DINGWALL, Robert. 2007. «Comment on the Presidential Address : Turn off the Oxygen... », Law and Society Review, vol. 41, n 4 : 787-795.

DUNEIER, Mitchell. 1999. Sidewalk. New York, Farrar, Strauss \& Giroux.

EHRENREICH, Barbara. 2001. Nickel and Dimed. On (not) Getting by in America. New York, Metropolitan books [trad. fr., L'Amérique pauvre. Comment ne pas survivre en travaillant. Paris, Grasset, 2004].

FASSIN, Didier. 2006. "The End of Ethnography as Collateral Damage of Ethical Regulation?», American Ethnologist, vol. 33, $\mathrm{n}^{\circ} 4$ : 522-524.

FEELEY, Malcolm M. 2007a. «Presidential Address : Legality, Social Research, and the Challenge of Institutional Review Boards », Law and Society Review, vol. 41, n 4 : 757-776.

— 2007b. «Response to Comments», Law et Society Review, vol. 41, n $4: 811-818$.

GofFman, Erving. 1961. Asylums. Essays on the Social Situation of Mental Patients and Other Inmates. Garden City (NY). Anchor books Doubleday.

GOLDMAN, Jerry J. et Martin D. KATZ. 1982. «Inconsistency and institutional review boards », Journal of the American Medical Association, vol. 248, $\mathrm{n}^{\circ} 2: 197-202$. 
GORDON, Elisa J. 2003. «Trials and Tribulations of Navigating IRBs : Anthropological and Biomedical Perspectives of "Risk" in Conducting Human Subjects Research », Anthropological Quarterly, vol. 76, n $2: 299-320$.

Gray, Bradford H., Robert A. CoOKE et Arnold S. TANnEnBAUM. 1978. « Research Involving Human Subjects: The Performance of Institutional Review Boards Is Assessed in this Empirical Study », Science, vol. 201, n 4361 : 1094-1101.

GreEnBERG, Daniel S. 1999. «When Institutional Review Boards Fail the System », The Lancet, vol. $353, \mathrm{n}^{\circ} 9166: 1773$.

— 2001. «Johns Hopkins Research Returns to Normal », The Lancet, vol. 358, n 9279 : 393.

HagGerTy, Kevin D. 2004. «Ethics Creep: Governing Social Science in the Name of Ethics », Qualitative Sociology, vol. 27, $\mathrm{n}^{\circ} 4$ : 391-414.

HANEY, Craig, William C. BANKS et Philip G. ZIMBARDO. 1973. «Interpersonal Dynamics in a Simulated Prison », International Journal of Criminology and Penology, $\mathrm{n}^{\circ} 1$ : 69-97.

HAYES, Gregory J., Steven C. HAYES, et Thane DYKSTRA. 1995. «A Survey of University Institutional Review Boards : Characteristics, Policies, and Procedures », IRB: Ethics and Human Research, vol. 17, $\mathrm{n}^{\circ}$ 3: 1-6.

Hine, Darlene C., William C. Hine et Stanley Harrold. 2000. The African-American Odyssey. Upper Saddle River (NJ), Prentice-Hall.

HoldEN, Constance. 1999. «Chancellor Quits After Research Shutdown », Science, vol. 285, $\mathrm{n}^{\circ} 5436$ : 2047.

HuMPHREYS, Laud. 2007 [1970]. Le commerce des pissotières. Pratiques homosexuelles anonymes dans l'Amérique des années 1960. Paris, La Découverte (Textes à l'appui), (éd. orig., Tearoom trade. Impersonal Sex in Public Places. Chicago, Aldine Publishing Company).

Jones, James H. 1981. Bad Blood. The Tuskegee Syphilis Experiment : A Tragedy of Race and Medicine. New York, The Free Press.

JOULE, Robert-Vincent et Jean-Léon BEAUVOIS. 1987. Petit traité de manipulation à l'usage des honnêtes gens. Grenoble, Presses universitaires de Grenoble (Vies sociales).

KATZ, Jack. 1988. Seductions of Crime. Moral and Sensual Attractions in Doing Evil. New York, Basic books.

— 2006. «Ethical Escape Routes for Underground Ethnographers », American Ethnologist, vol. 33, $\mathrm{n}^{\circ} 4$ : 499-506.

— 2007. «Comment on the Presidential Address: Toward a Natural History of Ethical Censorship », Law and Society Review, vol. 41, $\mathrm{n}^{\circ} 4$ : 797-810.

Kelman, Herbert C. 1968. A Time to Speak: On Human Values and Social Research. San Francisco, Jossey-Bass.

LEDERMAN, Rena. 2006. «The Perils of Working at Home : IRB Mission Creep as Context and Content for an Ethnography of Disciplinary Knowledges », American Ethnologist, vol. 33, n 4 : $482-491$.

Marshall, Patricia A. 1992a. «Anthropology and Bioethics », Medical Anthropology Quarterly, vol. 6, $\mathrm{n}^{\circ} 1: 49-73$.

- 1992b. «Research Ethics in Applied Anthropology », IRB: Ethics and Human Research, vol. 14, $\mathrm{n}^{\circ}$ $6: 1-5$.

- 2003. «Human Subjects Protections, Institutional Review Boards, and Cultural Anthropological Research », Anthropological Quarterly, vol. 76, n 2 : 269-285.

- 2004. «Human Subjects Protections, Institutional Review Boards, and Cultural Anthropological Research », Anthropological Quarterly, vol. 76, n 2 : 269-285.

MARWELL, Nicole P. 2007. Bargaining for Brooklyn. Community Organizations in the Entrepreunarial City. Chicago, University of Chicago Press.

MCDERMOTT, Monica. 2006. Working Class White. The Making and Unmaking of Race Relations. Berkeley, University of California Press.

MilgrAm, Stanley. 1994 [1969]. Soumission à l'autorité. Paris, Calmann-Lévy (Liberté de l'esprit), (éd. orig., Obedience to Authority : An Experimental View. New York, Harper Colophon Books).

NAtional Bioethics AdVisory COMMission. 2001. Ethical and Policy Issues in Research Involving Human Participants. Bethesda, MD, The Commission.

NAUDIER, Delphine. 2006. «Sociologie d'un miracle éditorial dans un contexte féministe », Genèses, $\mathrm{n}^{\circ}$ $64: 67-87$.

PAGER, Devah. 2002. «The Mark of a Criminal Record», PhD, Sociology, Madison, University of Wisconsin-Madison.

— 2003. «The Mark of a Criminal Record », American Journal of Sociology, vol. 108, n 5 : 937-975. 
PARK, Robert E. 2008. Le journaliste et le sociologue. Paris, Seuil-Presses de Sciences-po (Médiathèque) [Textes de 1923, 1940 et 1941 présentés et commentés par Géraldine Muhlmann et Edwy Plenel].

PlatTNER, Stuart. 2003. «Human Subjects Protection and Cultural Anthropology», Anthropological Quarterly, vol. 76, $\mathrm{n}^{\circ} 2: 287-297$.

PoOL, Ithiel de Sola. 1979. «Protecting Human Subjects of Research: An Analysis on Proposed Amendments to HEW Policy », Political Science and Politics, vol.12, n $4: 452-455$.

Rogers, Audrey S. et al. 1999. «A Case Study in Adolescent Participation in Clinical Research : Eleven Clinical Sites, One Common Protocol, and Eleven IRBs », IRB: Ethics and Human Research, vol. 21, ${ }^{\circ}$ $1: 6-10$.

Ross, Stephen L. et John YINGER. 2006. «Uncovering Discrimination : A Comparison of the Methods Used by Scholars and Civil Rights Enforcement Officials », American Law and Economics Review, vol. $8, \mathrm{n}^{\circ} 3: 562-614$.

Rothman, David J. 1982. «Were Tuskegee et Willowbrook "Studies in Nature" ? », Hastings Center Report, vol. $12, \mathrm{n}^{\circ} 2: 5-7$.

— et Sheila M. RothMAN. 1984. The Willowbrook Wars. New York, Harper \& Row.

SAVITT, Todd L. 1982. "The Use of Blacks for Medical Experimentation and Demonstration in the Old South », Journal of Southern History, vol. 48, n 3 : 331-348.

SHWEDER, Richard A. 2004. «Tuskegee re-examined», Spiked, consulté le 5 février 2008, http://www.spiked-online.com/Articles/0000000CA34A.htm

- 2006. «Protecting Human Subjects and Preserving Academic Freedom : Prospects at the University of Chicago », American Ethnologist, vol. 33, n 4 : 507-518.

SIEBER, Joan E., et Reuel M. BALUYOT, R. 1992. «A Survey of IRB Concerns about Social and Behavioral Research », IRB: Ethics and Human Research, vol. 14, n 2 : 9-10.

SILBERMAN, Roxane. 1999. Les sciences sociales et leurs données. Paris, La Documentation française.

Silverman, Henry, Sara C. Hull et Jeremy Sugarman. 2001. «Variability among Institutional Review Boards' Decisions within the Context of a Multicenter Trial », Crit Care Med, vol. 29, n 2 : 235-241.

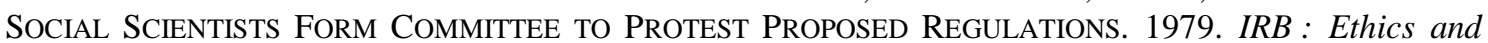
Human Research, vol. $1, \mathrm{n}^{\circ} 8: 7$.

STARK, Laura. 2006. «Morality in Science : How Research is Evaluated in the Age of Human Subjects Regulation », PhD, Sociology, Princeton, Princeton University.

- 2007. Victims in Our Own Minds ? IRBs in Myth and Practice, Law \& Society Review, vol. 41, n 4 : 777-786.

The NATIONAL COMmission fOR the PRotection of Human SubJects of BiomedicAL AND Behavioral ReSEARCH. 1979. The Belmont Report: Ethical Principles and Guidelines for the Protection of Human Subjects of Research. Washington, Department of Health, Education and Welfare. TROPP, Richard A. 1979. "What Problems Are Raised When the Current DHEW Regulation on Protection of Human Subjects Is Applied to Social Science Research ? », in The National Commission for the Protection of Human Subjects of Biomedical and Behavioral Research (éd.), Appendix to The Belmont Report: Ethical Principles and Guidelines for the Protection of Human Subjects of Research, vol. 2. Washington, Department of Health, Education, and Welfare : 18-1, 18-17.

— 1982. «A Regulatory Perspective on Social Science Research », in T. L. Beauchamp (éd.) : 391-415.

United States General ACCOUnTING OFFICE. 1996. Scientific Research : Continued Vigilance Critical to Protecting Human Subjects. Washington, report to the Ranking Minority Member, Committee on Governmental Affairs, US Senate.

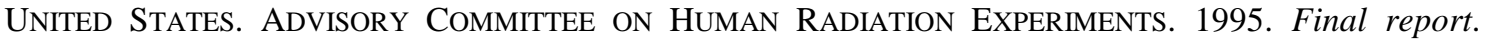
Washington, DC, Supt. of Docs., US GPO.

Venkatesh, Sudhir A. 2002a. American Project. The Rise and Fall of a Modern Ghetto. Cambridge (Mass.), Harvard University Press.

- 2002b. «Doin' the hustle. Contructing the Ethnographer in the American Ghetto », Ethnography, vol. $3, \mathrm{n}^{\circ} 1: 91-111$.

- 2006. Off the Books. The Underground Economy of the Urban Poor. Cambridge (Mass.), Harvard University Press.

- 2008. Gang Leader for a Day. A Rogue Sociologist Takes to the Streets. New York, The Pinguin Press.

WEBER, Florence. 2008. «Publier des cas ethnographiques : analyse sociologique, réputation et image de soi des enquêtés », Genèses, n 70 : 140-150.

ZIMBARdo, Philip G. 2007. The Lucifer Effect. Understanding How Good People Turn Evil. New York, Random House. 
i. http://www.afs-socio.fr/formCharte.html. Signalons également l'article de Daniel Céfaï (2009) dont nous avons eu connaissance trop tard pour l'intégrer à cet article.

ii. Cependant, le dernier rapport du Comité d'éthique en sciences sociales du CNRS (2007) indique que «ce dispositif n'a guère été appliqué » parce qu'il entraînerait «une lourdeur et une contrainte excessives», la loi n'étant pas adaptée aux recherches de sciences sociales qui ne présentent «aucun risque sérieux prévisible ».

iii. La récente charte de l'AFS prévoit la création d'un «Conseil de déontologie » au plan national et non local.

iv. Sur l'accès aux données quantitatives dans les pays développés, voir Silberman (1999). Les lois encadrant la collecte, l'archivage, l'usage et la transmission des données à caractère personnel ont été promulguées dans les démocraties occidentales à partir des années 1970. La loi «Informatique et Libertés » adoptée en France en 1978 a ouvert l'accès aux données pour des finalités administratives ou même de gestion par les opérateurs économiques, mais non de recherche. Le caractère restrictif de l'accès aux données quantitatives en France contraste avec la situation américaine, où le Freedom of Information Act de 1966 consacre le droit à l'information comme fondateur de la démocratie. La directive européenne 95/46/CE relative à la protection des données personnelles et à la libre circulation de ces données a expressément introduit les finalités de recherche, de statistique et d'histoire. Appliquée en 1998, elle n'a été transposée en France qu'en 2004 (loi du 6 août 2004 relative à la protection des personnes physiques à l'égard des traitements de données à caractère personnel).

v. Traduction de «United States Public Health Services».

vi. Le nombre exact de participants n'est pas clair. Selon D. C. Hine, W. C. Hine et S. Harrold (2000), il y a 431 malades et 191 bien portants; selon R. A. Shweder (2004), il y avait 410 malades (et un nombre inconnu de bien portants). La connaissance précise du nombre exact n'est pas cruciale pour l'argument.

vii. Le scandale de Willowbrook a connu un retentissement particulier parce qu'il a été alimenté par des détails scabreux : avant de leur injecter directement le virus, les médecins ont donné à manger aux enfants des extraits d'excréments d'humains malades de l'hépatite.

viii. Le serment d'Hippocrate et l'Introduction à l'étude de la médecine expérimentale de Claude Bernard (1865) interdisent explicitement au médecin de mener des expériences nuisibles aux patients, quel qu'en soit le bénéfice escompté pour le plus grand nombre.

ix. Dans l'expérience initiale, les sujets (les vrais cobayes) étaient tous des hommes ; quand l'expérience a été répliquée avec des femmes, les résultats ont été similaires ou avec des taux d'obéissance plus élevés. x. Voir notamment la préface d'Erving Goffman dans Asylums (1961).

xi. Il s'agit d'une traduction de « Department of Health, Education and Welfare », devenu Department of Health and Human Services en 1981, à la suite d'une tentative de Ronald Reagan de supprimer le ministère de l'Éducation.

xii. Le sénateur Edward Kennedy avait réuni en 1973 une commission d'enquête et, à la suite des auditions, introduit un projet de loi visant à protéger les sujets humains impliqués dans toutes les recherches financées par le gouvernement fédéral. Une agence indépendante devait en avoir la charge. Le projet de loi n'avait toutefois pas recueilli suffisamment de soutiens et E. Kennedy s'est replié sur les règles en cours d'élaboration au ministère de la Santé et des Affaires sociales. Ce faisant, le ministère n'a pas eu le temps de recueillir les contributions des services autres que le Service de santé publique (Tropp 1979, 1982).

xiii. Traduction de «National Commission for the Protection of Human Subjects of Biomedical and Behavioral Research ».

xiv. Le rapport tire son nom du centre de conférence de la Smithsonian Institution à Washington où les onze membres de la commission finalisent leur réflexion.

xv. Traduction de «Committee of Concern about IRB Practices».

xvi. Traduction de « National Bioethics Advisory Commission ».

xvii. Cette décentralisation complique également l'analyse du travail des IRB : dans un système décentralisé, les travaux qualitatifs encourent le risque de lister des anecdotes trop spécifiques et parfois caricaturales (De Vries et Forsberg 2002), tandis que les travaux quantitatifs agrègent des données issues de contextes locaux qui ont chacun leurs logiques de fonctionnement propres.

xviii. «Tout IRB doit comprendre au moins cinq membres, venus d'horizons différents, afin de promouvoir un examen complet et adapté des activités de recherche conduites couramment dans l'institution de recherche. L'IRB devra avoir les qualités requises en termes d'expérience, d'expertise et de diversité (rapport hommes femmes, diversité raciale et culturelle, appréciation des enjeux des 
différentes communautés) de façon à ce que ses conseils et avis en matière de protection des droits des sujets humains soient respectés » (arrêté 45CFR46).

xix. Les études décrivent également une surreprésentation des hommes blancs dans les IRB, ainsi que des universitaires (Hayes, Hayes et Dykstra 1995).

xx. On peut consulter Bourmeau (1988) et Park (2008) pour réfléchir sur les rapports entre sociologie (qualitative) et journaliste. Robert E. Park concevait le sociologue « comme une sorte de super-reporter, semblable à ceux qui écrivent dans Fortune (sic)» (Park 2008 : 38).

xxi. L'introduction à la psychologie sociale en langue française s'intitule par exemple Petit traité de manipulation à l'usage des honnêtes gens (Joule et Beauvois 1987).

xxii. Un drive-by shooting consiste à mitrailler sa victime en passant en voiture à côté d'elle puis à prendre la fuite. Cette méthode implique un risque élevé de dommages collatéraux.

xxiii. Sur les questions éthiques relatives à l'anonymat, voir Weber (2008).

xxiv. Les IRB délivrent une approbation valable sur une durée déterminée. Dans le cas où la recherche doit être prolongée, le chercheur doit demander une extension de l'approbation, nécessitant un examen de la recherche en cours. 